\title{
Electrochemical Behaviour of Lotus-Type Porous SUS304L and SUS316L Stainless Steels
}

\author{
Minoru Fuseya ${ }^{1}$, Takuji Nakahata ${ }^{1}$, Soong-Keun Hyun ${ }^{1, *}$, \\ Shinji Fujimoto ${ }^{2}$ and Hideo Nakajima ${ }^{1}$ \\ ${ }^{1}$ The Institute of Scientific and Industrial Research, Osaka University, Ibaraki 567-0047, Japan \\ ${ }^{2}$ Division of Materials and Manufacturing Science, Graduate School of Engineering, \\ Osaka University, Suita 565-0871, Japan
}

Lotus-type porous metals are expected to be used in various applications such as lightweight structural materials and biomedical materials. Lotus-type porous stainless steel is particularly promising as a structural material because stainless steel has useful properties such as high corrosion resistance, high workability, low cost and so on. However, there is a possibility that dissolved hydrogen or the microstructure of lotustype porous stainless steel affects its corrosion behaviour. In this study, the electrochemical corrosion behaviour of lotus-type porous SUS304L and SUS316L stainless steels fabricated by the continuous zone melting technique under pressurized hydrogen was investigated using a potentiodynamic polarization in $0.1-\mathrm{kmol} / \mathrm{m}^{3}$ sulphuric acid solution. The current density of lotus-type porous SUS304L was higher than that of nonporous SUS304L at around $-100 \mathrm{mV}(-100 \mathrm{mV}$ peak), while it was similar to that of nonporous stainless steel in the passive and transpassive regions. The specific current peak observed for the porous SUS304L at around $-100 \mathrm{mV}$ disappears when the pores are filled with epoxy resin or the specimens are dehydrogenated. Thus, it is concluded that the $-100 \mathrm{mV}$ peak is attributed to the dissolved hydrogen at pore surface. In the case of lotus-type porous SUS316L, corrosion behaviour is similar to that of SUS304L. [doi:10.2320/matertrans.47.2229]

(Received February 28, 2006; Accepted July 27, 2006; Published September 15, 2006)

Keywords: corrosion, polarization curve, porous metal, stainless steel

\section{Introduction}

Recently, porous metallic materials have attracted considerable attention due to their potential practical use as lightweight materials, catalyst supports and electrodes with large specific surface areas, vibration and acoustic energy damping materials, impact energy absorption materials, etc. Extensive studies have been done on fabrication of porous copper, iron, nickel, magnesium and their alloys by the Czochralski method or by the unidirectional solidification method under pressurized hydrogen or nitrogen atmosphere. ${ }^{1-10)}$ These metals and alloys have many cylindrical pores which are elongated in the solidification direction and have recently been referred to lotus-type porous metals because their structure looks like lotus root. Usually, conventional porous metals comprise spherical pores and exhibit almost isotropic properties. Owing to the elongated pore shape of lotus-type porous metals, they exhibit various anisotropic behaviour and also good fluid permeability and high strength in the pore elongated direction. ${ }^{6-10)}$ Therefore, they have been developed for practical use ${ }^{11)}$ as face plates of golf clubs, artificial bones, etc. However, their corrosion behaviour, which is important for their practical application, has not been systematically examined.

Stainless steel is one of the most important alloys with a high corrosion resistance because of the Cr-enriched stable passivity. Moreover, it is well known for its high workability and low cost. ${ }^{12)}$ Therefore, lotus-type porous stainless steel is particularly promising as lightweight and high-corrosionresistance structural material. However, the corrosion behaviour of lotus-type porous stainless steel may be affected by its microstructure or the dissolved hydrogen. In the present study, the corrosion behaviour of lotus-type porous

*Corresponding author, E-mail: hyun23@sanken.osaka-u.ac.jp stainless steel was investigated by the electrochemical method. SUS304L and SUS316L were selected as test samples because they are widely used and representative of austenite stainless steel.

\section{Experimental Procedure}

Lotus-type porous SUS304L and SUS316 stainless steels were fabricated by the continuous zone melting technique under pressurized hydrogen atmosphere. Figure 1 shows a schematic drawing of this technique. A high-frequency induction heating coil and a pair of upper and lower sample rod holders, which can be moved in the vertical direction, are placed in a chamber. Ambient hydrogen is blown on the part of the sample rod below the coil in order to enhance the cooling rate. The pore diameter and porosity can be controlled by changing the gas pressure and the transference

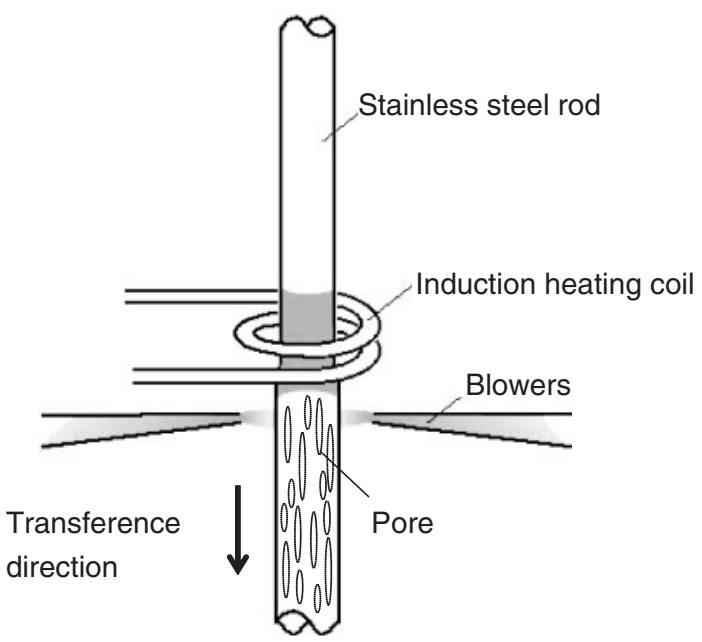

Fig. 1 Schematic drawing of continuous zone melting technique. 
Table 1 Fabrication conditions for porous and nonporous stainless steel.

\begin{tabular}{lcc}
\hline & $\begin{array}{c}\text { Atmosphere, } \\
p / \mathrm{MPa}\end{array}$ & $\begin{array}{c}\text { Transference velocity } \\
\text { of rod, } v / \mu \mathrm{m} \mathrm{s}^{-1}\end{array}$ \\
\hline Porous SUS304L & $1.0\left(\mathrm{H}_{2}\right)$ & 160 \\
Nonporous SUS304L & $1.0(\mathrm{He})$ & 500 \\
Porous SUS316L & $2.0\left(\mathrm{H}_{2}\right)$ & 160 \\
Nonporous SUS316L & $2.0(\mathrm{He})$ & 160 \\
\hline
\end{tabular}

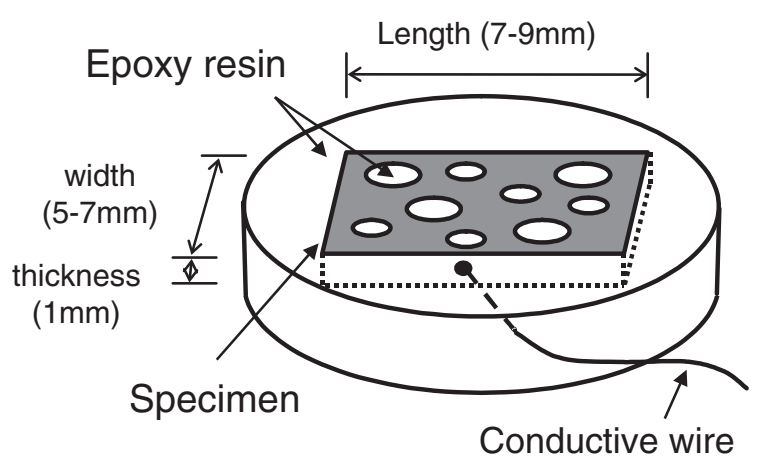

Fig. 2 Schematic drawing of porous stainless steel embedded in an epoxy resin.

velocity of the rod. ${ }^{9,10)}$ The transference velocities of the rod and the gas pressures for porous and nonporous SUS304L and SUS316L are listed in Table 1. Plate-shaped specimens of $1 \mathrm{~mm}$ in thickness, 5-7 mm in width and 7-9 $\mathrm{mm}$ in length cut out of the ingots using a spark-erosion wire-cutting machine (A320D, Sodick, Ltd.) and polished using No. 800 $\mathrm{SiC}$ paper.

Additionally, reference specimens were prepared. In order to investigate the effect of the pore surface on the corrosion behaviour, porous SUS304L and SUS316L were embedded in an epoxy resin to fill the pores, as shown in Fig. 2. Furthermore, dehydrogenated specimens were prepared in order to investigate the effect of dissolved hydrogen. SUS304L and SUS316L were dehydrogenated at $473 \mathrm{~K}$ under a vacuum of $2.0 \times 10^{-3} \mathrm{~Pa}$ for $3.6 \mathrm{ks}$.

The porosity, average pore diameter and pore perimeter of the specimens were measured by an image analysis system (Macscope, Mitani Corp). Considering that all the pores penetrated the specimen, the superficial area $S_{A l l}$ can be estimated by the porosity $\varepsilon$ and the pore perimeter as follows:

$$
S_{A l l}=2\{w \times l \times(1-\varepsilon)+(w+l) \times t\}+t \times c,
$$

where $w$ is the width, $l$ is the length, $t$ is the thickness of the specimen and $c$ is the integrated value of the pore perimeter.

Figure 3 shows a schematic of the polarization curve measurement system. This system consisted of an electrochemical cell that comprised a conventional three-electrode configuration with a platinum wire and a $\mathrm{Ag} / \mathrm{AgCl}$ electrode as the counter and reference electrodes, respectively. The potentiodynamic polarization curve ranging from the corrosion potential to $1500 \mathrm{mV}$ was measured by a potentiostat (PS-07, Toho Giken Co. Ltd.). Henceforth, the electrode potential will be described with respect to this reference electrode. The scanning rate was $1 \mathrm{mV} / \mathrm{s}$. Prior to the measurement, an electrolytic solution of $0.1 \mathrm{kmol} / \mathrm{m}^{3} \mathrm{H}_{2} \mathrm{SO}_{4}$

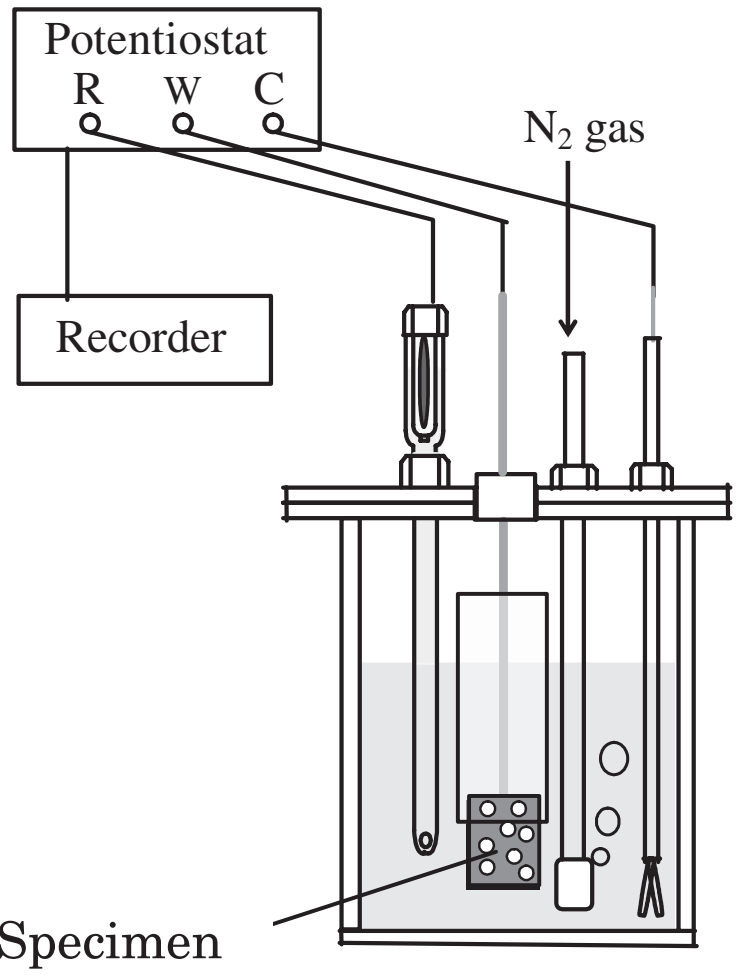

Fig. 3 Schematic drawing of polarization curve measurement system.

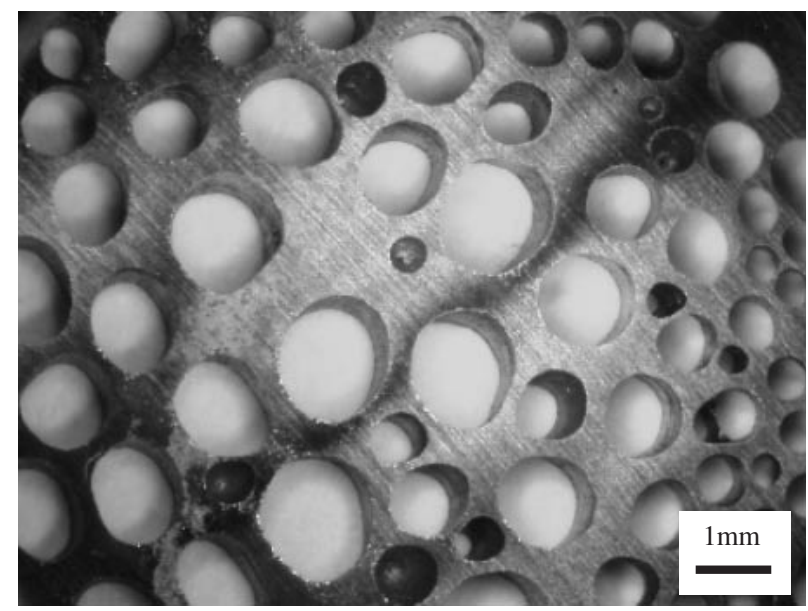

Fig. 4 Cross-sectional planes perpendicular to the pore-growth direction.

was deaerated by bubbling high-purity nitrogen for $30 \mathrm{~min}$.

The structures of the specimens were analyzed by an X-ray diffractometer (RIGAKU AFC-7R) using $\mathrm{Cu} \mathrm{K} \alpha$ radiation. In order to measure the amount of hydrogen remaining in the specimens, the hydrogen concentration was analyzed using a hydrogen determinator (EF400, LECO Co.). Before the measurement, each specimen was finely cut into an open pore.

\section{Results and Discussion}

\subsection{SUS304L}

A cross-sectional plane perpendicular to the pore-growth direction of porous SUS304L is shown in Fig. 4. The porosity and average pore diameter are $55.8 \%$ and $1126 \mu \mathrm{m}$, respec- 


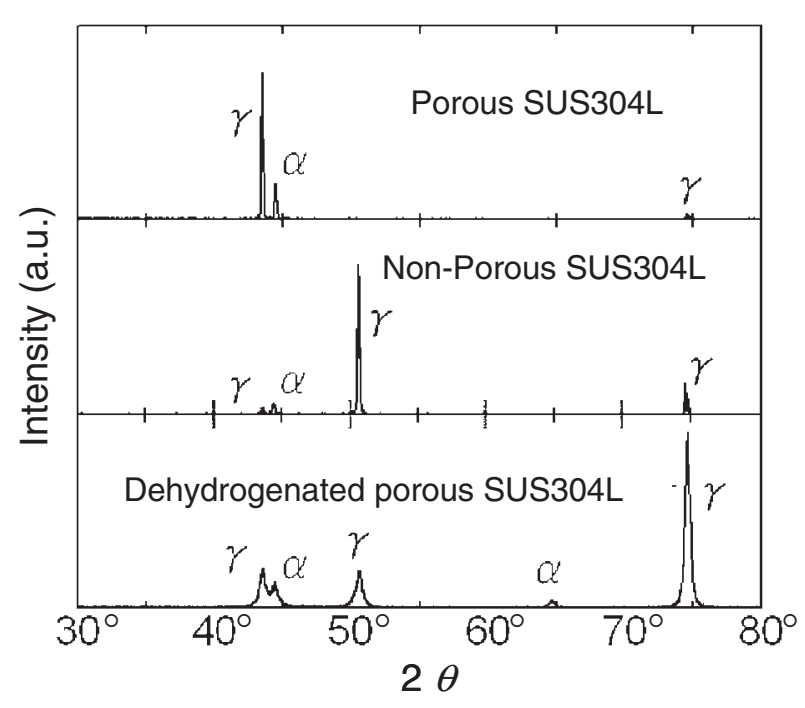

Fig. 5 XRD spectra of nonporous and porous SUS304L.

Table 2 Hydrogen concentration of porous SUS304L.

\begin{tabular}{lc}
\hline & $\begin{array}{c}\text { Hydrogen concentration, } \\
x / \text { mass ppm }\end{array}$ \\
\hline Starting material & 7.3 \\
(commercial SUS304L) & 22.1 \\
Porous SUS304L & 6.1 \\
Dehydrogenated porous SUS304L & \\
\hline
\end{tabular}

tively. It is evident that most of the pores penetrate the 1-mmthick specimen. The XRD spectra of nonporous and porous SUS304L (as-cast and dehydrogenated) are shown in Fig. 5. In comparison with nonporous SUS304L, porous SUS304L exhibits prominent peaks associated with the $\alpha$ phase. The stable phases of SUS304L are the $\gamma$ phase at high temperature and the $\alpha+\gamma$ phase at room temperature; ${ }^{12)}$ further, for the fabrication in the present study, the transference velocity of the rod for porous SUS304L was lower than that for nonporous SUS304L. Thus, it is considered that the $\alpha+\gamma$ phase appeared in the porous SUS304L specimen because its cooling rate was insufficient to form a single $\gamma$ phase. Moreover, the peaks associated with the $\alpha$ phase were also observed in the dehydrogenated specimen. It is found that the microstructure of the lotus-type porous SUS304L did not change due to heat treatment at $473 \mathrm{~K}$ for $3.6 \mathrm{ks}$.

The hydrogen concentration of porous SUS304L is listed in Table 2. Due to dehydrogenation, the dissolved hydrogen in porous SUS304L decreased to the similar concentration to that of the starting material.

The potentiodynamic polarization curves are shown in Fig. 6. The current density in the passive region, which ranges from 200 to $800 \mathrm{mV}$, for both porous and nonporous SUS304L is almost the same. SUS304L forms a very stable and $\mathrm{Cr}$-enriched passive film in the passive region because of the selective dissolution of $\mathrm{Fe}$ in aqueous solution. On the other hand, a specific current peak was observed for the porous SUS304L at around $-100 \mathrm{mV}$. In this potential region, the current density of embedded porous SUS304L was lower than that of porous SUS304L and similar to that of

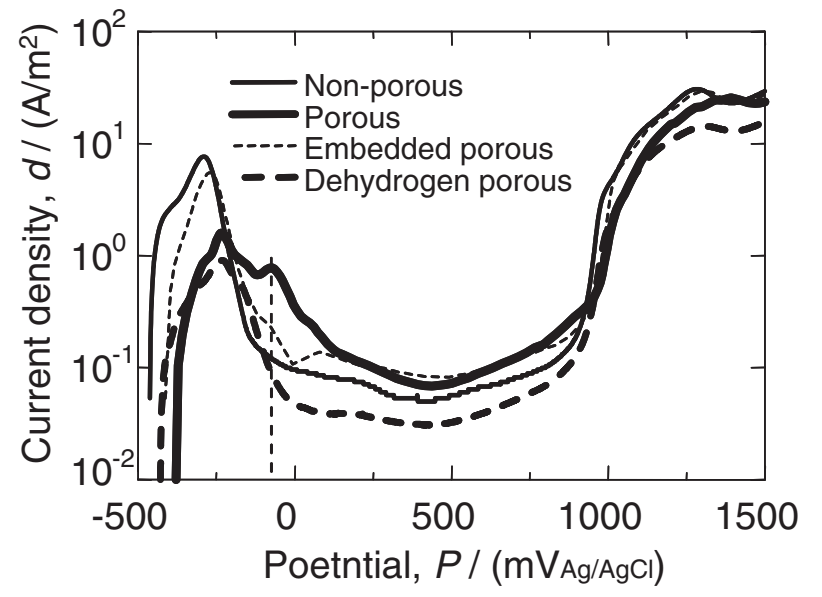

Fig. 6 Potentiodynamic polarization curves of SUS304L stainless steel in deaerated $0.1 \mathrm{kmol} \cdot \mathrm{m}^{-3} \mathrm{H}_{2} \mathrm{SO}_{4}$.

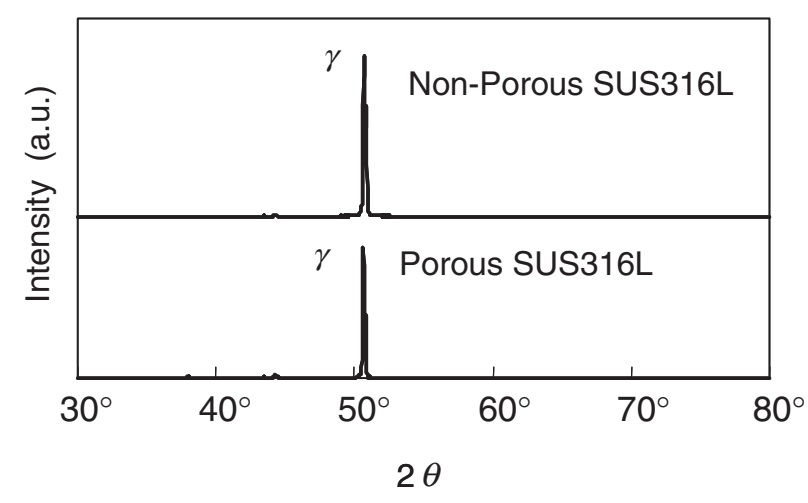

Fig. 7 XRD spectra of nonporous and porous SUS316L.

nonporous SUS304L. In other words, it is revealed that the corrosion behaviour on the pore surface is significantly different from that in the bulk area. It was also found that the current density of dehydrogenated porous SUS304L was low and almost similar to that of nonporous SUS304L.

At the active peak, which ranges from the corrosion potential to $-200 \mathrm{mV}$, the lower current densities were observed for porous SUS304L and dehydrogenated porous SUS304L than those of non porous SUS304L and embedded SUS304L. The pore surface of porous SUS304L, both with and without dehydrogenation, was never polished; however, it was covered with air-formed oxide films that suppress active dissolution. Therefore, porous specimens showed a lower active peak current.

On the other hand, although the structure of porous SUS304L possessed the $\alpha$ phase, the current density of embedded porous SUS304L was similar to that of nonporous SUS304L over the entire region. Therefore, it may be concluded that the $\alpha$ phase in as-cast lotus-type porous SUS304L does not affect the corrosion behaviour.

\subsection{SUS316L}

The porosity and average pore diameter of SUS316 were $42.2 \%$ and $834 \mu \mathrm{m}$, respectively. The XRD spectra of nonporous and porous SUS316L are shown in Fig. 7. In both the spectra, only the peak associated with the $\gamma$ phase 


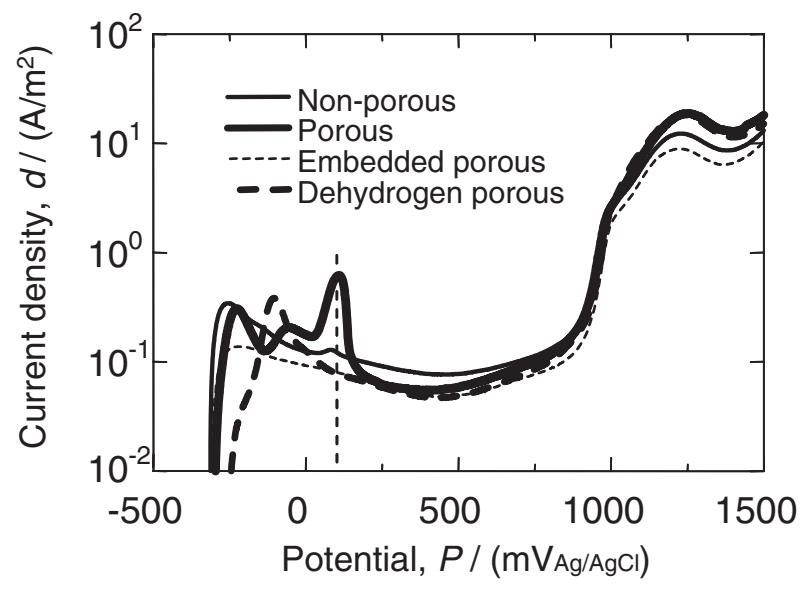

Fig. 8 Potentiodynamic polarization curves of SUS316L stainless steel in deaerated $0.1 \mathrm{kmol} \cdot \mathrm{m}^{-3} \mathrm{H}_{2} \mathrm{SO}_{4}$.

was observed. In contrast with SUS304L, the stable phase of SUS316L is the $\gamma$ phase at room temperature. ${ }^{12)}$

The potentiodynamic polarization curves of SUS316L are shown in Fig. 8. The current density in the passive region was almost similar for all the specimens examined. However, a specific current peak was observed at around $100 \mathrm{mV}$ for porous SUS316L; this peak is similar to that of dehydrogenated porous SUS304L at around $-100 \mathrm{mV}$. In this region, the current density of embedded porous SUS316L was lower than that of porous SUS316L and similar to that of nonporous SUS316L. Moreover, the current density of dehydrogenated porous SUS316L was comparable to that of nonporous SUS316L.

\subsection{Role of hydrogen and pore on the electrochemical behaviour}

Similar polarization curves were observed for non-porous, embedded porous and also as-received specimens of SUS304L and SUS316L. It means that the polished bulk materials exhibit similar polarization behaviour. Therefore, it is concluded that specific microstructure, including $\alpha$ phase observed in SUS304L, and solid-dissolved hydrogen does not bring about significant change in the electrochemical property. On the other hand, the inside of the pore behaves different dependence on the specimen condition. As described before, the active dissolution was suppressed by the air formed oxide, which could be not removed by the cleaning process prior to measurements. The specific current peak located in the transition from active to passive region should be correlated with hydrogen at pore surface, because it is confirmed that the specific peaks never appear for bulk material independent on hydrogen content.

Moreover, the specific current peaks observed for lotustype porous stainless steels disappeared after dehydrogenate heat treatment or pre-dissolution of pore surface by electropolishing. Therefore, it is highly possible that the specific current increase in the polarization curves is correlated with some surface state generated during solidification. Formation of stable passive film was hindered by hydride or some chemical state on the pore surface. The hindrance could disappear at transition potential region to form a stable passive film with some anodic dissolution. Alternatively, when such surface compound might be oxidized to dissolve or transform to stable passive film, an anodic current appeared.

Although, in the present work, analysis on the surface of pores was not conducted, it is concluded that some chemical states correlated with hydrogen are formed on the pore surface and the formation of stable passive film may be hindered. Such harmful property of lotus-type porous stainless steels fabricated in hydrogen atmosphere could be easily eliminated by dissolution of pore surface or dehydrogenation.

\section{Conclusions}

The corrosion behaviour of lotus-type porous SUS304L and SUS316L is similar to that of their nonporous counterparts in the passive region. However, it was found that the pore surfaces of both porous SUS304L and SUS316L reveal a peculiar current increase at around -100 and $100 \mathrm{mV}$, respectively; this increase could be eliminated by dehydrogenation. The dissolved hydrogen hinders the formation of a stable passive film. On the other hand, if the air-formed oxide film on the pore surface is not removed, it suppresses dissolution in the active potential region.

\section{Acknowledgements}

The present work was supported by Grant-in-Aid for the Creation of Innovations through Business-Academic-Public Sector Cooperation of the Ministry of Education, Culture, Sports, Science and Technology of Japan. This work was also supported by the Priority Assistance of the Formation of Worldwide Renowned Centers of Research and the 21st Century COE Program (Project: Center of Excellence for Advanced Structural and Functional Materials Design) from the Ministry of Education, Culture, Sports, Science and Technology of Japan.

\section{REFERENCES}

1) S. Yamamura, H. Shiota, K. Murakami and H. Nakajima: Mat. Sci. Eng. A 318 (2001) 137-143.

2) S. K. Hyun and H. Nakajima: Mater. Trans. 43 (2002) 526-531.

3) T. Ikeda and H. Nakajima: J. Jpn. Foundry Eng. Soc. 74 (2002) 812816.

4) T. Ikeda, M. Tsukamoto and H. Nakajima: Mater. Trans. 43 (2002) 2678-2684.

5) J. Banhart and D. Weaire: Phys. Today 55 (2002) 37-42.

6) H. Nakajima, S. K. Hyun and T. Ikeda: Acta Technica Napocensis 45 (2002) 3-6.

7) S. K. Hyun, K. Murakami and H. Nakajima: Mater. Sci. Eng. A 299 (2001) 241-248.

8) S. K. Hyun and H. Nakajima: Mater. Sci. Eng. A 340 (2003) 258-264.

9) T. Ikeda, T. Aoki and H. Nakajima: Bull. Iron Steel Inst. Japan 90 (2004) 9-16.

10) T. Ikeda, T. Aoki and H. Nakajima: Metal. Mater. Trans. 36A (2005) 77-86.

11) H. Nakajima: J. Soc. Powder Technol. Japan 40 (2003) 108-114.

12) M. Hasegawa: Stainless Steel Handbook (The Nikkan Kougyo Shimbun, Japan 1960). 